\title{
A review: Prediction model for the incidence rate of intracerebral hemorrhage
}

\author{
Kaikai Yin ${ }^{1, a}$, Le Zhang ${ }^{1, ~ b *}$, Hua Feng ${ }^{2, c}$, Rong $\mathrm{Hu}^{2, \mathrm{~d}}$ \\ ${ }^{1}$ Southwest university, Chongqing, China \\ ${ }^{2}$ Third Military Medical University, Chongqing, China \\ ayinkai0828@swu.edu.cn \\ bzhanglcq@swu.edu.cn \\ cfenghua8888@vip.163.com \\ dhuchrong@aliyun.com
}

Keywords:Intracerebral hemorrhage; artificial intelligence; Computed tomography angiography (CTA)

\begin{abstract}
Intracerebral hemorrhage (ICH) accounts for around 10-30\% incidence of the cerebral apoplexy, the prognosis of which is so poor that has high morbidity and mortality. In cerebral hemorrhage, the artery bleeding is very common. Along with the development of imaging, computed tomography angiography (CTA) can be used to predict the incidence of intracerebral hemorrhage. Based on the previous studies for ICH. This study proposed such a novel mathematical model for the incidence of cerebral hemorrhage prediction that not only investigated the potential risk factors $\mathrm{ICH}$, but also employed CTA images and statistic test to explore which potential risk factors can be used as the classifier of the mathematical model. Next, we used training data set to train the weight of these selected risk factors by artificial intelligence algorithm as well as employed testing data to validate the predictive power of the model. We expected this mathematical model can be be able to predict the incidence rate of cerebral hemorrhage and offer the corresponding preventive suggestion in the distant future.
\end{abstract}

\section{Introduction}

Intracerebral hemorrhage (ICH) is an acute, spontaneous bleeding from the cerebral parenchymal blood vessels. Intracerebral hemorrhage accounts for $10 \%$ to $30 \%$ of cases of acute cerebral apoplexy. The case fatality rate of patients with intracerebral hemorrhage within one month is as high as 30\% 50\%[1] and more than $30 \%$ of survivors survive with neurological dysfunction. Though the drug for cerebral hemorrhage and high blood pressure can significantly control the risk of the cerebral hemorrhage incidence, the clinical consequences of cerebral hemorrhage is very serious and the mortality rate of the patients with cerebral hemorrhage is still as high as 50\%[2, 3]. However, the pre-diagnosis for ICH can effectively reduce the incidence rate of ICH $[4,5]$. Therefore, this study purposes to develop such a mathematical model that can predict the incidence probability of ICH.

Currently, there are a lot of studies for the intracerebral hemorrhage pre-diagnosis. Especially, the research on the application of information technology for predicting the risk of cerebral hemorrhage incidence is comprised of two directions. One is to predict the incidence rate of intracerebral hemorrhage[3, 6-8]. For example, Capon et al.,[6] investigated the relationship between the seasons and cerebral hemorrhage, which demonstrated that the temperature could be used to predict the risk of cerebral hemorrhage. Ciccone et al.,[7] reported that the gender also affected the incidence rate of intracerebral hemorrhage. However, the predictive object of these research was based on a special group of people rather than the individual patient. And several important properties of the cerebral hemorrhage (such as blood 
pressure) were not considered in the predictive model. The other direction is to predict ICH hematoma expansion[1, 5, 8-20]. For example, Wada et al.,[2] build up a prediction model by CTA "spot sign", which can predict hematoma expansion for individual patients. Nonetheless, CTA spot sign can't be applied to predict the probability of intracerebral hemorrhage directly as well as the model does not use training data to optimize its key parameters.

To overcome the shortcomings of the previous research $[1-10,13]$, this study put forward an improved algorithm to predict ICH. First, a set of statistical tests will be used to validate which potential risk factors suggested by the clinical personnel are statistically significant. And then, these validated risk factors will be employed as the classifiers to develop the predictive model. Next, artificial intelligence algorithm will be used to train the key parameters of the predictive model by fitting the simulated results against the training experimental data as well as using testing data compute the predictive power of the predictive model.

\section{Marterial and Pre-research methods}

Computed tomography angiography (CTA) is a rapid, noninvasive investigation for patients with ICH and it is useful for identifying potentially treatable entities such as aneurysms and other vascular lesions , which provides help and guidance for further clinical treatment [2]. The CTA images of this study come from the Third Military Medical University. The poor quality of the CTA images are excluded in the data preprocessing stage.

This research put forward a mathematical model (Fig. 1) to predict the incidence rate of intracerebral hemorrhage, which consists of three steps: (1) Data preprocessing, (2) feature selection and (3) model training and testing.

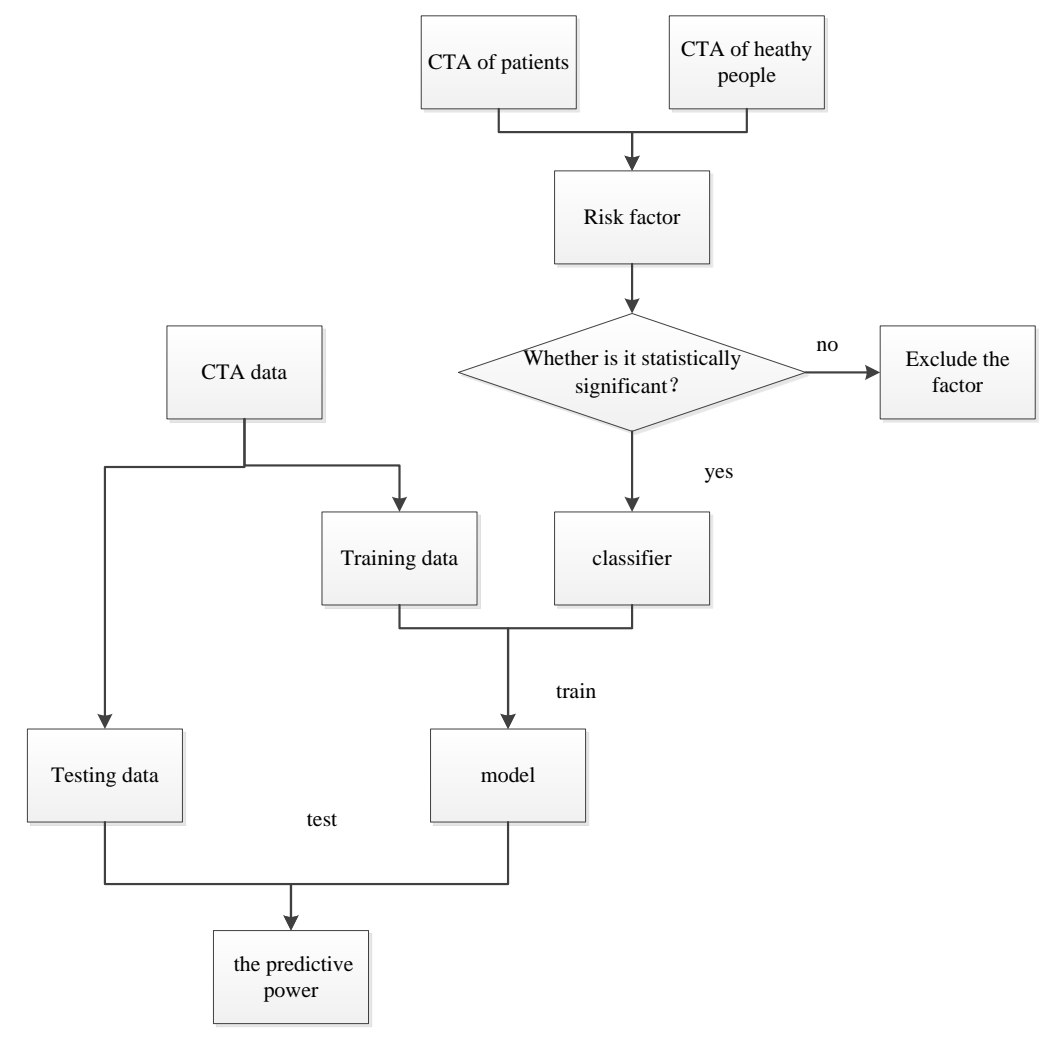

Fig1. The workflow chart 


\subsection{Data preprocessing}

The rupture of lenticulostriate arteries is commonly occurred in intracerebral hemorrhage[21]. The diameter of the lenticulostriate arteries is tiny, generally less than 0.5 millimeter. Since not all of lenticulostriate arteries can be identified clearly in the CTA image, we have to choose the CTA images with clear lenticulostriate arteries from source CTA image set (Fig.2). Also, we sharpen the raw CTA image by Laplace sharpening method[22] to improve the image quality. Fig 3 shows that the sharpened CTA image is better than the original image (Fig. 2). Finally, we reconstructed CTA images to have Fig.4, in which the lenticulostriate arteries are circled too.



Fig 2. The raw CTA image and the red cvcle indicates the Lenticulostriate arteries



Fig 3. The CTA image after Laplace sharpening

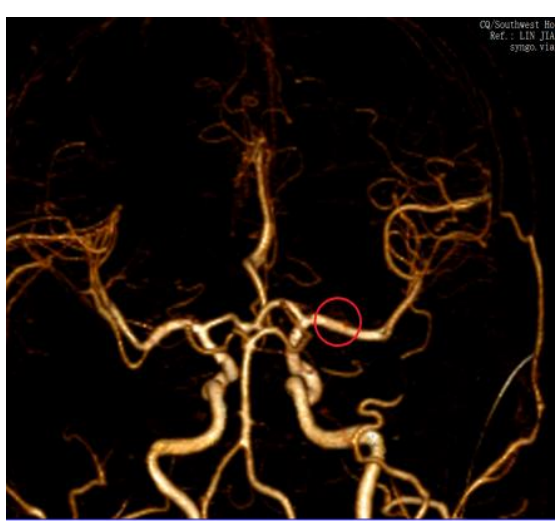

Fig 4. Lenticulostriate arteries indicated by red cycle in the CTA reconstructed image

\subsection{Feature selection}

We chose the potential risk factors provided by the clinical personnel (such as glucose levels, blood pressure, the use of antihypertensive medication, blood vessel diameter, blood flow, and so on). And we extracted the values of these potential risk factors from the CTA images (such as diameter, density, area and so on). After that, a set of statistical tests are used to validate which features are statistically significant. Lastly, these statistically significant features are employed as the classifiers to develop the predictive model.

\subsection{Model training and testing}

After we selected the statistically significant features, we planed to employ several classical data mining algorithm such as decision tree[23], Bayes network[24] and supporting vector machine[25] to develop such a mathematical model that can predict the incidence probability of ICH. Before starting the model development, we will divide the CTA image set into the training and the testing data sets. The training data set will be employed to train the weight of the classifier of our predictive model. And the testing data set will be used to validate the predictive power of the mathematical model. Finally, sensitive analysis will be carried out to explore which parameters are the key parameters of the model.

\section{Expected results and discussion}

By the analysis of the human brain CTA Images, this study expects to build up such a mathematical model to predict the incidence probability of ICH. Data preprocessing expected to improve the CTA image resolution by the digital image processing and reconstructed CTA images for the further research. Feature selection employed statistic test methods to explore which risk features are statistically significant. Model training and testing step employed training data and data mining algorithm to optimize the key parameters of the model as well as used testing data to validate the predictive power of the model. Because of the high mortality and disability rate of cerebral hemorrhage, it is very important to pre-diagnosis and prevent intracerebral hemorrhage. Therefore, we expect to establish such a sufficiently accurate forecasting model 
with respect to the artificial intelligence theory and CTA images that can not only predict the incidence of cerebral hemorrhage, but also provide the corresponding preventive suggestions.

\section{Acknowledgment}

This work supported by the Natural Science Foundation of China under Grant No. 61372138, Chongqing excellent youth award and the Chinese Recruitment Program of Global Youth Experts, as well as by Fundamental Research Funds for the Central Universities No. XDJK2014B012 and NO. XDJK2016A003

\section{References}

[1] J. Broderick, H. Adams, W, W. Feinberg, E. Feldmann, J. Grotta, C. Kase, et al., "Guidelines for the management of spontaneous intracerebral hemorrhage: A statement for healthcare professionals from a special writing group of the Stroke Council, American Heart Association," Stroke, vol. 30, pp. 905-915, 1999.

[2] R. Wada, R. I. Aviv, A. J. Fox, D. J. Sahlas, D. J. Gladstone, G. Tomlinson, et al., "CT angiography "spot sign" predicts hematoma expansion in acute intracerebral hemorrhage," Stroke; a journal of cerebral circulation, vol. 38, pp. 1257-62, 2007.

[3] R. Leira, ., A. Dávalos, ., Y. Silva, ., A. Gil-Peralta, ., J. Tejada, ., M. Garcia, ., et al., "Early neurologic deterioration in intracerebral hemorrhage Predictors and associated factors," Neurology, vol. 63, pp. 461-7, 2004.

[4] E. F. Chang, M. Michele, and M. C. Holland, "Acute traumatic intraparenchymal hemorrhage: risk factors for progression in the early post-injury period," Neurosurgery, vol. 61, pp. 647-656, 2006.

[5] V. A. Coenen, S. Dammert, M. H. T. Reinges, M. Mull, J. M. Gilsbach, and V. Rohde, "Imageguided microneurosurgical management of small cerebral arteriovenous malformations: the value of navigated computed tomographic angiography," Neuroradiology, vol. 47, pp. 66-72, 2005.

[6] A. Capon, G. Demeurisse, and L. Zheng, "Seasonal variation of cerebral hemorrhage in 236 consecutive cases in Brussels," Stroke; a journal of cerebral circulation, vol. 23, pp. 24-27, 1992.

[7] A. Ciccone, M. Pozzi, C. Motto, P. Tiraboschi, and R. Sterzi, "Epidemiological, clinical, and therapeutic aspects of primary intracerebral hemorrhage," Neurological Sciences Official Journal of the Italian Neurological Society \& of the Italian Society of Clinical Neurophysiology, vol. 29 suppl 2, pp. 256-257, 2008.

[8] C. T. Skidmore and A. John, "Spontaneous intracerebral hemorrhage: epidemiology, pathophysiology, and medical management," Neurosurgery Clinics of North America, vol. 13, pp. 281-288, 2002.

[9] P. C. Sanelli, M. J. Mifsud, and P. E. Stieg, "Role of CT angiography in guiding management decisions of newly diagnosed and residual arteriovenous malformations," Ajr American Journal of Roentgenology, vol. 183, pp. 1123-1126, 2004.

[10] J. Kim, W. Smith, Y. Lu, W. Dillon, and M. Wintermark, "Contrast extravasation on CT predicts mortality in primary intracerebral hemorrhage," Ajnr American Journal of Neuroradiology, vol. 29, pp. 520-525, 2008.

[11] S. C. Chin, C. H. Chang, T. Y. Chang, K. L. Huang, T. C. Wu, J. R. Lin, et al., "Brain computed tomography perfusion may help to detect hemodynamic reconstitution and predict intracerebral hemorrhage after carotid stenting," Journal of vascular surgery: official publication, the Society for Vascular Surgery [and] International Society for Cardiovascular Surgery, North American Chapter, vol. 56, pp. 1281-1290, 2012. 
[12] K. Lars, S. Marek, G. Christoph, H. Oliver, and P. A. Ringleb, "Blood pressure variability after intravenous thrombolysis in acute stroke does not predict intracerebral hemorrhage but poor outcome," Cerebrovascular Diseases, vol. 33, pp. 135-140, 2012.

[13] K. Becker, A. Baxter, Hm, D. Tirschwell, T. Abouelsaad, and W. Cohen, "Extravasation of radiographic contrast is an independent predictor of death in primary intracerebral hemorrhage," Stroke; a journal of cerebral circulation, vol. 30, pp. 2025-2032, 1999.

[14] J. M. Wardlaw, "Prediction of haematoma expansion with the CTA spot sign: a useful biomarker?," Lancet Neurology, vol. 11, pp. 294-295, 2012.

[15] A. Ederies, A. Demchuk, T. Chia, D. J. Gladstone, D. Dowlatshahi, G. Bendavit, et al., "Postcontrast CT extravasation is associated with hematoma expansion in CTA spot negative patients," Stroke; $a$ journal of cerebral circulation, vol. 40, pp. 1672-6, 2009.

[16] S. Gazzola, R. I. Aviv, D. J. Gladstone, G. Mallia, V. Li, A. J. Fox, et al., "Vascular and nonvascular mimics of the CT angiography "spot sign" in patients with secondary intracerebral hemorrhage," Stroke; a journal of cerebral circulation, vol. 39, pp. 1177-1183, 2008.

[17] J. E. Delgado Almandoz, H. R. Kelly, P. W. Schaefer, H. B. Brouwers, A. J. Yoo, M. J. Stone, et al., "CT angiography spot sign predicts in-hospital mortality in patients with secondary intracerebral hemorrhage," Journal of Neurointerventional Surgery, vol. 4, pp. 442-7, 2012.

[18] H. B. Brouwers, G. J. Falcone, K. A. Mcnamara, A. M. Ayres, A. Oleinik, K. Schwab, et al., "CTA Spot Sign Predicts Hematoma Expansion in Patients with Delayed Presentation After Intracerebral Hemorrhage," Neurocritical Care, vol. 17, pp. 421-428, 2012.

[19] A. M. Demchuk, D. Dowlatshahi, D. Rodriguez-Luna, C. A. Molina, Y. S. Blas, I. Dzialowski, et al., "Prediction of haematoma growth and outcome in patients with intracerebral haemorrhage using the CT-angiography spot sign (PREDICT): a prospective observational study," Lancet Neurology, vol. 11, pp. 307-314, 2012.

[20] D. Dowlatshahi, B. Brouwers, A. Demchuk, M. D. Hill, R. Aviv, L. A. Ufholz, et al., "Abstract 114: The Predictive Ability of the CTA Spot Sign for Hematoma Expansion is Dependent on Time Since ICH Onset: A Systematic Review and Patient-level Meta-analysis," Stroke, p. A114, 2013.

[21] K. Hyun-Seung, H. Moon Hee, K. Bae Ju, K. O-Ki, K. Sung Hyun, and C. Kee-Hyun, "Evaluation of the lenticulostriate arteries with rotational angiography and 3D reconstruction," in American Journal of Neuroradiology, 2005, pp. 306--312.

[22] L. U. Yun-Wei and Y. R. Chen, "The Research and Realization of Image Sharpening Based on Laplace," Computer Knowledge \& Technology, 2009.

[23] J. R. Quinlan, "Induction on decision tree," Machine Learning, vol. 3, pp. 351-360, 1986.

[24] G. Dan, "Bayesian Network Classifiers," in Machine Learning, 1997, pp. 131--163.

[25] B. Huang, "Prediction of blood-brain barrier penetrating drugs using Supporting Vector Machine," Computers \& Applied Chemistry, vol. 26, pp. 188-190, 2009. 